\title{
Method of the Year 2008
}

\author{
With its tremendous potential for understanding cellular biology now poised to \\ become a reality, super-resolution fluorescence microscopy is our choice for Method \\ of the Year.
}

Like it or not, 2008 is at an end. At Nature Methods, in our annual celebration of the techniques that drive biological research and of the people who develop them, it is time to pick our Method of the Year.

As electron microscopy did in the past, superresolution microscopy, or nanoscopy, provides the ability to see details of cellular and even macromolecular structure that were not possible to see before. Notably, however, nanoscopy is compatible with live cells and has the capability for multiplex labeling with high molecular specificity. This exciting prospect has driven our choice of nanoscopy as Method of the Year 2008.

For centuries, people have been using the light microscope to look at structures too small to see with the eye alone, and light microscopes in their myriad forms are now a vital fixture in the laboratory. Despite great strides in microscope technology over the years, however, the so-called diffraction barrier, which dictates a limit on the resolution that can be achieved by a light microscope, was considered inviolable.

Recent work conducted largely by physicists in interdisciplinary academic settings shows that this fallacy - though rooted in theory — can be laid to rest. These researchers have devised multiple ways to realize nanometer-scale resolution in fluorescence microscopy. The technical developments, though slow at first, have recently reached a fever pitch.

In a Primer on page 19, we outline the basic principles underlying the most common techniques for achieving super-resolution, and in a technical Perspective on page 24, Stefan Hell, the originator of this revolution, discusses the present and future implementation of nanoscopy methods in depth.

As described in a News Feature on page 15, development of the approaches that make present-day nanoscopy possible began in the 1990s. Though nanoscopy has been implemented in cells for over a decade, this has so far largely taken the form of proof-ofprinciple demonstrations that the techniques work in the cellular context. We are convinced that nanoscopy is now poised to make the transition into widespread biological application.

Like last year, we include also a selection of Methods to Watch (p.33), a subjective and necessarily incomplete list of approaches that, although they may not as yet have come entirely into their own, are likely to develop in interesting and fruitful ways in the coming years.

As part of the selection process this year, we wanted to include a reader's choice, and had asked you for nominations of exciting methods and for votes on these nominations. We thank those of you who took the time to participate and are particularly pleased to see that several of your choices overlapped with our own selections for this year's Methods to Watch. But the number of votes we received was insufficient for us to truly gauge our readership's opinion as a whole. We will begin the voting earlier the next time around!

Last year, we picked next-generation sequencing as Method of the Year, and we thought that this year's presentation would be incomplete without a brief look back at how the technology has performed since. Already relatively proven at the time, the applicability and utility of 'next-gen' sequencing both to additional whole-genome sequencing and to functional genomics was clear. Indeed, in 2008, next-gen sequencing has largely lived up to its promise. To mention just a few highlights, we have seen this year the resequencing of entire worm and human genomes, the sequencebased profiling of the transcriptome and of noncoding RNAs in several organisms, and the mapping of cytosine methylation sites in the genomes of mammals and of plants.

We predict that nanoscopy, although still taking baby steps in its application to biology, will make for an equally exciting year ahead. As discussed in more detail in a Commentary by Jennifer Lippincott-Schwartz on page 21 , although it is critical that the technology is used with cognizance of its strengths and its weaknesses, there are multiple areas of cell biology — the study of cellular architecture or of molecular heterogeneity, for instance - that stand to gain immensely from being visible at the nanometer scale.

Furthermore, it is entirely likely that there are things to see inside of living cells that we have not as yet been able to imagine. Life at the nanometer scale could be a whole new world.

In the meantime, visit our website and watch the movie! 